\title{
Smart Building Research Based on BIM
}

\author{
JinxuLi \\ Chongqing Vocational Institute of Engineering, Chongqing, China, 402260
}

\begin{abstract}
Smart building is the inevitable trend of future architectural development. This paper first introduces the concept of smart building, and then gives the concept and characteristics of BIM. Using these characteristics of BIM technology, we can effectively improve the safety, energy-saving, efficiency and comfortableness to create a more smart building.
\end{abstract}

Keywords: smart building, BIM, energy-saving

\section{Concept of Smart Buildings}

Smart building combines the construction equipment, office automation and communication network, optimization structure, service and management as a system to provide people with a safe, efficient, comfortable and convenient building environment. The construction of smart building integration is not wisdom, but important technical methods and techniques to realize the wisdom of building security, efficient, convenient and comfortable working and living environment, its intelligence services in the construction of social benefits, economic benefits and environmental benefits, not for the integrated and integrated, intelligent integration is just a means not the final aim. The wisdom of building life cycle information management and construction as the theoretical basis, the management information system as the means, through the building and outside the building, data collection from people, architecture, environment, and data processing and analysis, the formation of valuable information to help building managers to make decisions, so as to improve the energy and the equipment operation efficiency, reduce the construction cost management, and 
ultimately improve the people and buildings, the relations between architecture and environment, make it become more harmonious. The ultimate manifestation of smart building is a systematic and highly integrated information management system. For the construction of information technology, because of its information management technology and the logic of optimization is no higher requirements, it is more suitable for grafting a set of information management system on it. We only need to provide input to the building related data, the system can feedback the relevant results. The IT process is completely in the middle of the black box. This approach is more suitable as the basis of the concept of intelligent architecture and the realization of specific functions. Smart building is produced in the Internet of things, big dataand cloud computing and smart city development under the background. It will not only achieve the sustainable development of green building, but also realize the cooperation of all information on the same platform.

\section{Concept and Features of BIM}

\subsection{Concept}

Building information model (BIM) is based on the 3D digital technology. It integrates the engineering data construction project related information model is a digital expression of the facilities of the project's physical and functional characteristics. BIM is the core of a construction project includes the professional and the various stages of the database, the database can be shared and used for the project parties, each party can accord the permissions to modify and expand the relevant content in the database. Early hand-painted drawings are onedimensional or two-dimensional drawings expression. It needs to be divided into complete drawing plans, elevations and sections of a side view, making drawings become abstract and complicated, always need to introduce a lot of repeat information caused low efficiency. The information of the building model is very large and the structure is complex, the traditional drawing technology cannot collect all the information of the building, and cannot analyze and judge the whole and the progress of the project accurately. BIM technology for building information model, whole process and data of building design, construction, operation and maintenance of the object of analysis and description, each unit involved in the construction of all can use the data and resources.

\subsection{Features}

In the current environment of architectural design, architectural drawings of each component in the drawings used only to draw the lines of expression, despite the effect graph modelling software to make can make people have a sense of the appearance of the building, but related to the configuration and system of pipelines cannot directly in the form of three-dimensional presentation, need professional combination the plane drawings to imagine. It includes not only the three-dimensional model of the building, but also the three-dimensional model of 
the plumbing and heating equipment, etc., at any stage of the design, we can see the design effect of each part at any time. The two-dimensional drawing drawings never carries on the analysis and Simulation of intelligent computing, often need to separate modelling in the related analysis software, which will spend a lot of time, and will make the calculation model of rough error. The use of information technology to create BIM model contains all the data, so the model will guide people to the analysis based on the technology of BIM simulation software, you can analyse the structure, thermal performance analysis, pipeline inspection, fire safety inspection, the conflict of energy analysis, specification test. Not only saves time, but also ensures the accuracy of the data. As the building information model contains all the information in the building, so the construction of various types of work can be based on this model for collaborative design.For the construction industry, the use of visualization of the real role in the construction industry is very large, for example, often get the construction drawings, but the various components of the information in the drawings of the line drawing expression, but its true form requires building industry participants to imagine. For simple things, it is also not a bad idea, but in recent years the construction industry construction forms, complex shapes constantly launch, so the light by the brain to imagine what would be impractical. So BIM provides visual ideas, so that people will type the member lines form a three-dimensional real 3D graphic display in front of people; the construction industry also has a design renderings of things, but this effect diagram is subcontracted to renderings of professional production team for line type design information literacy made out of production, and not through the automatic generation of component information, the lack of isomorphism between interactive and visual feedback, however BIM mentioned is an interactive and visual feedback of the form of isomorphism between BIM in building information model, the whole process is visible so, to generate visual results. It not only can be used to display and report the effect chart, more importantly, can be used in the project design, construction and operation.

\section{Applications of BIM in the Smart Buildings}

\subsection{Enhance security of smart buildings}

The safety of the building is the primary indicator of smart building. BIM based on a large number of real data for various types of precision design, effectively improve the safety of smart buildings. The statistics and calculation of the number of traditional building components and equipment are carried out by manpower. The quality of the staff has a great impact on the accuracy of the work. With the BIM model provides highly accurate basic data, efficient and accurate material statistics possible. Under the premise of perfect data, the material statistics can be extracted from the BIM model database by the special software function. Due to the characteristics of high-speed computer operation, the efficiency and accuracy of statistical work has been improved. The use of virtual construction technology of BIM model, the equipment and materials construction step using the prior analysis, and in some factory equipment and facilities, reduce 
local processing and processing at the site link, which can not only reduce the space occupied, inventory and processing personnel, also can ensure the quality, speed up the construction of facilities. Another important application of BIM technology in the design phase is collision detection. BIM collision detection refers to the functional integrity of the building model and quality standards for testing, generally including whether there is overlap components, structural stress is reasonable, whether in line with the laws and regulations standards. First, the $3 \mathrm{D}$ model is established firstly, and then the target of collision detection is needed. The collision detection is carried out, and the final conclusion is obtained after the modification. The use of BIM simulation technology, collision detection, can be found in the design of the deficiencies, to optimize the improvement, but also to avoid the construction process because of the design loopholes.

\subsection{Decrease consumption of smart buildings}

The layout and design of the building should be conducive to the summer ventilation and winter warmth, the thermal performance of building envelope should reach the reference value and design value. The reference value can be chosen to be similar to the designed building, the structure is similar, the size is similar to the same building. Once the simulated building energy consumption exceeds the reference energy consumption of the building, it is necessary to adjust the thermal performance parameters of the building envelope, so as to achieve the required energy saving effect. The advantage of BIM technology is that it can quickly calculate the parameters, greatly reduce the time required by the artificial statistics, and the data of the simulation output has a high degree of credibility. It can be analyzed directly to the building indoor sunshine, sunshine and light shadow simulation of emission factors that impact on the environment of BIM can automatically calculate the parameters of the building shape coefficient, comprehensive specific data of architectural design, energy-saving design and energy saving design, according to the Standard Specification for automatic checking; BIM software can analyze the dynamic energy consumption that, the cost of building energy-saving and non-energy saving ratio, help designers choose reasonable insulation system. The application of BIM technology saves a lot of materials, reducing costs. Green building materials should be as far as possible to choose renewable and recyclable materials, in the absence of pollution and safety of the premise of the use of recycled materials should be more than $10 \%$ of the total amount of building materials. The construction unit shall provide a recyclable material summary sheet and the proportion of the calculation table, these parameters are input to the BIM software using material list module, calculation type and weight of recycled materials, so as to judge whether the architectural design can meet the requirements of energy-saving design.

\subsection{Promote efficiency of smart buildings}

Only the efficient building can be called the smart building. Based on the data provided by the BIM model, the BIM project management software should be 
able to pass the four-dimensional space correlation, rapid and accurate calculation of engineering quantity, improve the accuracy and efficiency of the construction budget due to BIM data accuracy of the component level, can quickly provide support and management of the line required for the project data, effectively improve the efficiency of construction management. The fundamental reason for the meticulous management of construction enterprises is difficult to achieve because of the huge amount of engineering data, which cannot be obtained quickly and accurately in order to support resource planning. The emergence of BIM can quickly and accurately obtain basic engineering data for relevant management line, to provide effective support for the construction enterprise to make accurate, material and material plan, greatly reducing the waste of resources of logistics and warehousing sectors, in order to realize the limits of consumption, to provide technical support for control. Through the statistical analysis of the BIM model, we can directly and accurately get the workload of each day or even every hour in the construction plan and the quantity of material and equipment. The function of $3 \mathrm{D}$ visualization BIM model plus the time dimension, can carry out virtual construction, the construction plan quickly whenever and wherever possible direct comparison with actual progress at the same time, effective coordination, construction, supervision and non-engineering industry background of project owners led to various problems and aware of the situation. Through the BIM technology combined with the Construction Scheme Simulation and on-site video monitoring, we can greatly improve the degree of coordination in the construction process, reasonable distribution of the working interface and working procedures. This can not only effectively reduce the construction quality problems and reduce the rework and rectification, but also can improve the construction efficiency, shorten the construction cycle, effectively improve efficiency.

\subsection{Strengthen comfortableness of smart buildings}

The use of glass curtain wall building curtain wall is a mirror or glass curtain wall, outdoor landscape lighting illumination can directly into the air, building lighting effects on the surrounding buildings, can be simulated and analyzed by BIM software. First by the construction side to provide special report of the construction plan, design specification and architectural drawings of the building, and then by the third party test lighting and light pollution analysis and compile analysis report, the sunshine analog building lighting effects generated report to the special information and test data input BIM software, optimize the environment of the building ventilation room and also can be simulated and analyzed by BIM software to design the content according to the report, the design wind speed of indoor air circulation and pedestrian areas should be kept in a comfortable range. We will enter the above information into the BIM software for wind environment analysis, the results of wind speed, such as the need to improve the timely reminder and optimization. We now know that PMV can be used to replace the temperature to measure the thermal comfort of the environment. This has a great effect on saving energy. For example, if you can 
increase the speed to reduce the PMV value to a comfortable range. It can avoid open air conditioning more power, further saving appliances use energy consumption. That is to say, we can use different control strategies to meet the requirements of thermal comfort. In contrast to the past, only air conditioning to regulate the temperature. Such thinking will be more energy-efficient space. The principle is that the choice of the most energy-efficient equipment to achieve the most comfortable thermal environment. Compared with the thermal environment on the air conditioning control system, BIM technology will refer to the indoor environment, providing comfortable control strategy for the users.

\section{Conclusion}

BIM technology provides an important technical platform for the development of smart building, which is conducive to the sustainable development of the construction industry. The application of BIM technology to the design and construction of smart building is the necessary way for the development of China's construction industry. Currently, BIM technology is not perfect and the application of smart building BIM is still in its infancy. But we believe that in the near future, BIM technology will promote great progress of China's smart building industry.

\section{References}

[1] Wang Yuqing, Mu Huaqian, Application of BIM Technology inSmart buildings, Electrical Technology of Smart buildings, 10(5), pp. 53-57, 2016.

[2]Jia Shuang, Shen Xiaowei, Zhang Baogang, On application of green building design based on BIM technique, 42(10), pp. 209-210, 2016.

[3] Li Nong, Liu Xuanye, The Prospects of Applying BIM Technology to Architectural Lighting Design, China Illuminating Engineering Journal, 24(5), pp. 12-15+63, 2013

[4] Gong Yue, Fang Jun, Lean Cooperation Development of New Building IndustrializationBased on the BIM Technology, Construction Technology, 45(18), pp. 38-42, 2016. 